\title{
UNIFORM DOMAINS AND CAPACITY
}

\author{
STEPHEN M. BUCKLEY AND DAVID A. HERRON
}

\begin{abstract}
We characterize the class of uniform domains in terms of capacity. As a byproduct of this investigation we provide results describing when a Loewner domain will be QED.
\end{abstract}

\section{INTRODUCTION}

The importance of the class of uniform domains in Euclidean space and their role in geometric analysis is well established. Uniform domains were first studied by John [Joh61] and Martio and Sarvas [MS79] and their significance in function theory is well documented; see [Geh87], [Väi88]. Every (bounded) Lipschitz domain is uniform, but there are also uniform domains with fractal boundary. More recently, generalizations of this concept to domains in Heisenberg and other Carnot groups have become a focus of study; cf. [CT95], [CGN00], [?]. See Section 2 for basic information including precise definitions, notation and terminology.

In this paper, we prove the equivalence of (a)-(c) of the following theorem; the equivalence of (a) and (d) is proven in [BH05]. Relevant definitions are given below in Section 2.

1.1. Theorem. For a domain $D \subsetneq \mathbf{R}^{n}$, the following are quantitatively equivalent.

(a) $D$ is a uniform domain.

(b) $D$ is QED with respect to Whitney balls and a Gromov domain.

(c) $D$ is Loewner and a k-cap domain.

(d) $D$ is $L L C$ with respect to arcs, quasiconvex, and a weak slice domain.

The phrase 'quantitatively equivalent' means that the various parameters associated with the stated conditions depend only on each other and the dimension $n$. It is easy to see that uniform domains possess all of the properties indicated above, so the significance here is that these various conditions are sufficient for uniformity. That condition (b) implies uniformity is a generalization of [HK96, Theorem A]; see Theorem 3.5. That (c) implies (a) follows from Theorem 3.10, which in turn provides some information regarding the question as to whether or not every Loewner domain in $\mathbf{R}^{n}$ is actually QED.

The equivalence of (a) and (d) is included above because (d) is in some ways related to conditions (b) and (c). However, unlike the capacitary conditions in (b) and (c), both conditions (a) and (d) make sense in the more general metric space setting, and we prove their equivalence in this context in[BH05]. In the current paper, we examine only uniform and capacitary conditions in Euclidean space $\mathbf{R}^{n}$.

One notable corollary of Theorem 1.1 is the following; again, we prove only the equivalence of (a), (b), (c) in this paper, and leave the equivalence of (a) and (d) for [BH05].

Date: 13 December 2005.

1991 Mathematics Subject Classification. Primary: 30C62; Secondary: 30C20, 30F45, 28A80.

The first author was supported by Enterprise Ireland. The second author was supported in part by the Charles Phelps Taft Memorial Fund. 
1.2. Corollary. Suppose $D=G \backslash E$, where $G \subsetneq \mathbf{R}^{n}$ is a uniform domain and $E$ is a closed set contained in $G$ which is removable for the Sobolev space $W^{1, n}$. The following are equivalent:

(a) $D$ is a uniform domain.

(b) $D$ is a Gromov domain.

(c) $D$ is a k-cap domain.

(d) D is a weak slice domain.

The class of $Q E D$ domains $D \subset \mathbf{R}^{n}$, which satisfy a quasiextremal distance inequality

$$
\bmod (E, F ; D) \geq C^{-1} \bmod \left(E, F ; \mathbf{R}^{n}\right)
$$

for all disjoint continua $E, F$ in $D$, was introduced by Gehring and Martio [GM85] and has subsequently been studied by many authors. We examine these, along with weaker variants such as our $\mathrm{QED}_{\mathrm{b}}$ and $\psi$-QED conditions. The class of Loewner domains is a special case of the class of Loewner spaces introduced by Heinonen and Koskela in their study [HK98] of quasiconformal mappings of metric spaces; Heinonen's recent monograph [Hei01] renders an enlightening account of these ideas.

Every QED domain, and more generally every $\psi$-QED domain, is a Loewner domain. The question of whether or not a Loewner domain is always QED appears to be open. We prove the following two theorems in this direction. The first, a consequence of Theorem 3.10 and Proposition 3.7, says that the Loewner property always implies two weaker versions of QED, while the second result lists some classes of domains for which Loewner implies the full-strength QED condition. Precise definitions are given in $\S 3$.A and $\S 3$.B; for now we just mention that, in the terminology of [HK91], a Euclidean domain has no large and no small boundary components if the diameters of the components $C$ of the boundary, $C \neq\{\infty\}$, are bounded away from both zero and infinity.

1.3. Theorem. A Loewner domain $D \subsetneq \mathbf{R}^{n}$ is both $Q E D_{b}$ and $\psi-Q E D$, quantitatively.

1.4. Theorem. A Loewner domain $D \subsetneq \mathbf{R}^{n}$ is $Q E D$ in each of the following instances:

(a) $D$ is a Gromov domain.

(b) $\mathbf{R}^{n} \backslash D$ has n-measure zero.

(c) $n=2$ and there is a quasiconformal self-homeomorphism of $\mathbf{R}^{2}$ mapping $D$ to a domain having no large and no small boundary components.

The QED condition can be weakened in two ways. First, we can assume that the defining inequality holds only for a smaller class of disjoint continua, such as closed balls or closed Whitney balls. Second, we can replace the right-hand side of the inequality by $\psi\left(\operatorname{cap}\left(E, F ; \mathbf{R}^{n}\right)\right)$ where $\psi:[0, \infty) \rightarrow[0, \infty)$ is some homeomorphism; we then talk of a $\psi$-QED condition. It is known that the QED condition for balls does not imply the full QED condition [HK96, Example 4.1]. Nevertheless, we show that QED for Whitney balls implies QED for all balls; this provides an answer to Question 8.5 in [HK96]. In fact we have the following more general result.

1.5. Theorem. If $D \subsetneq \mathbf{R}^{n}$ is $\psi-Q E D$ with respect to Whitney balls, then it is $\psi^{\prime}-Q E D$ with respect to

all balls, where $\psi^{\prime}(t)=c \psi(c t)$ for $0<t<1 / 2$ and $\psi^{\prime}(t)=$ ct for $t \geq 1$, and $c>0$ depends only on $n$ and the Whitney ball constant.

A remarkable feature of the above result is that $\psi^{\prime}$ is linear for $t \geq 1$ even if $\psi$ grows much more slowly. 
This document is organized as follows: Section 2 contains preliminary information including basic definitions and terminology descriptions as well as elementary and previously known facts. The bulk of our contributions are presented in Section 3. We introduce the notion of $\psi$-QED domains in $\S 3$.A and demonstrate that $\psi$-QED ${ }_{\mathrm{wb}}$ domains are $\psi^{\prime}-\mathrm{QED}_{\mathrm{b}}$ (see Theorem 3.3); this useful fact also answers Question 8.5 in [HK96]. We turn our attention to Loewner domains in $\S 3 . \mathrm{B}$ and establish results relating these to $\psi$-QED domains. We provide proofs of the results stated above in $§ 3 . C$. Finally, in Section 4 we exhibit examples related to our main results.

\section{Preliminaries}

2.A. General Information. Our notation is relatively standard. We write $C=C(a, \ldots)$ to indicate a constant $C$ which depends only on the parameters $a, \ldots$; the notation $A \lesssim B$ and $B \gtrsim A$ both mean that there exists a constant $c$ with $A \leq c B$, and $A \simeq B$ means that $A \lesssim B$ and $B \lesssim A$ both hold. Typically $a, b, c, C, K, \ldots$ will be constants that depend on various parameters, and we try to make this as clear as possible, often giving explicit values. However, at times $C$ will denote some constant whose value depends only on the data present but may differ even on the same line of inequalities.

Throughout this article we work in Euclidean space $\mathbf{R}^{n}, D$ is a domain (i.e., an open connected set) in $\mathbf{R}^{n}$, and $d(x, y):=|x-y|$ is Euclidean distance. However, see $\S 2$.G. We denote by $B(x ; r):=\{y:|x-y|<r\}$ and $S(x ; r):=\partial B(x ; r)=\{y:|x-y|=r\}$ the open ball and sphere of radius $r$ centered at $x \in \mathbf{R}^{n}$, and write $t B(x ; r):=B(x ; t r)$ for $t>0$. When working in a given domain $D \subsetneq \mathbf{R}^{n}$, we let $d(x)=\operatorname{dist}(x, \partial D)$ be the Euclidean distance from a point $x \in D$ to the boundary $\partial D$ of $D$; also, we write $B(x):=B(x ; d(x))$ and, given $\lambda \in(0,1)$, we call $\lambda B(x)=B(x ; \lambda d(x))$ a Whitney ball at $x$ with parameter $\lambda$.

The constants $\Omega_{n}, \omega_{n-1}$, and $\sigma_{n}$ respectively, stand for the $n$-measure of the unit ball $\mathbf{B}^{n}=B(0 ; 1)$, the $(n-1)$-measure of the unit sphere $\partial \mathbf{B}^{n}$, and the $n$-dimensional sphericalcap constant respectively.

An arc is the homeomorphic image of an interval, and it is open or closed if the interval is open or closed, respectively. When $x, y$ are points on an arc $\gamma$ we denote by $\gamma[x, y]$ the subarc of $\gamma$ between $x$ and $y$ (with endpoints included). If $\gamma$ is merely a path, then $\gamma[x, y]$ refers to a fixed but arbitrary subpath of $\gamma$ with included endpoints $x, y$. We denote by $\Gamma(x, y ; D)$, or simply $\Gamma(x, y)$, the family of all rectifiable paths in $D$ from $x$ to $y$ (with endpoints included).

2.B. Quasihyperbolic Distance. The quasihyperbolic distance for a domain $D \subsetneq \mathbf{R}^{n}$ is defined by

$$
k(x, y)=k_{D}(x, y):=\inf _{\gamma \in \Gamma(x, y ; D)} \ell_{k}(\gamma):=\inf _{\gamma \in \Gamma(x, y ; D)} \int_{\gamma} \frac{d s}{d(z)},
$$

where $d s$ is the Euclidean arclength element. This infimum is always achieved and the minimal quasihyperbolic length paths are called quasihyperbolic geodesics. In fact, thanks to the Hopf-Rinow theorem, we know that the metric space $(D, k)$, which we call the quasihyperbolization of $D$, is always a proper geodesic space.

We remind the reader of the following basic estimates for quasihyperbolic distance, first established by Gehring and Palka [GP76, 2.1]:

$$
k(x, y) \geq \log \left(1+\frac{l(x, y)}{d(x) \wedge d(y)}\right) \geq j(x, y):=\log \left(1+\frac{|x-y|}{d(x) \wedge d(y)}\right) \geq\left|\log \frac{d(x)}{d(y)}\right|
$$


See also [BHK01, (2.3),(2.4)]. The first inequality above is a special case of the more general (and easily proven) inequality,

$$
\ell_{k}(\gamma) \geq \log \left(1+\frac{\ell(\gamma)}{d(x) \wedge d(y)}\right) \quad \text { for all } \gamma \in \Gamma(x, y)
$$

Following [BHK01, Chapter 7], we call a proper subdomain $D$ of $\mathbf{R}^{n}$ a Gromov domain if its quasihyperbolization $(D, k)$ is a Gromov hyperbolic metric space, i.e., if all quasihyperbolic geodesic triangles are $\Delta$-thin for some $\Delta>0$, which means that each point on an edge of any such triangle is within distance $\Delta$ of some point on one of the other two edges. The interested reader should consult the references in [BHK01] and [BB03] for information concerning Gromov hyperbolicity. Let us note that uniform domains are Gromov domains and Gromov hyperbolicity is preserved by quasiconformal mappings; in particular, all finitely connected plane domains $D \subsetneq \mathbf{R}^{2}$ are Gromov.

2.C. Uniform Domains. Given $x, y \in D \subsetneq \mathbf{R}^{n}$, an arc $\gamma \in \Gamma(x, y ; D)$ is an a-uniform arc, $a \geq 1$, provided that $\ell(\gamma) \leq a|x-y|$ and

$$
\min \{\ell(\gamma[x, z]), \ell(\gamma[y, z])\} \leq \operatorname{ad}(z) \quad \text { for all } z \in \gamma
$$

We call $\gamma$ a double a-cone arc if it satisfies the inequality displayed above (the phrases cigar arc and corkscrew are also used). A domain $D$ is a-uniform if each pair of points in $D$ can be joined by an $a$-uniform arc.

An important characterization of uniformity is due to Gehring and Osgood [GO79, Theorems 1,2], who proved that uniform domains are precisely those domains in which the quasihyperbolic distance is bilipschitz equivalent to the $j$-distance defined in $\S 2$.B. For our purposes we require the following seemingly weaker sufficient condition for uniformity; this result is due to Väisälä [Väi10, 6.16, 6.17]. Here

$$
r(x, y)=r_{D}(x, y):=\frac{|x-y|}{d(x) \wedge d(y)}
$$

denotes the so-called relative distance between $x, y \in D$.

2.1. Fact. Let $D \subsetneq \mathbf{R}^{n}$ be a domain. Then $D$ is uniform if and only if there is a homeomorphism $\vartheta:[0, \infty) \rightarrow[0, \infty)$, with $\lim \sup _{t \rightarrow \infty} \vartheta(t) / t<1$, such that for all points $x, y \in D$, $k(x, y) \leq \vartheta(r(x, y))$. The uniformity constant depends only on $\vartheta$, and conversely when $D$ is a-uniform, one can always take $\vartheta(t)=b \log (1+t)$ with $b=b(a)$. Moreover, the estimate $k(x, y) \leq \vartheta(r(x, y))$ for $k(x, y) \geq 2$ is also equivalent to uniformity (since such an estimate for small quasihyperbolic distance holds in all domains).

2.D. Modulus and Capacity. The conformal modulus (or $n$-modulus) of a family $\Gamma$ of curves in a metric measure space $(X, d, \mu)$ is

$$
\bmod \Gamma=\inf \int \rho^{n} d \mu
$$

where the infimum is taken over all $\Gamma$-admissible densities, meaning all Borel functions $\rho: X \rightarrow[0, \infty]$ satisfying $\int_{\gamma} \rho d s \geq 1$ for all locally rectifiable curves $\gamma \in \Gamma$. Here $n$ is the Hausdorff dimension of $X$. Given a pair of disjoint compact sets $E, F \subset X$, we let $(E, F ; X)$ be the family of all curves joining the sets $E, F$ in $X$. We also let $\Gamma(E, F ; X)$ denote the family of all rectifiable curves in $(E, F ; X)$. The quantity $\bmod (E, F ; X)$ is called the conformal modulus (of the condensor $(E, F, X)$ ). 
The conformal, variational, or $n$-capacity of a pair of disjoint, compact sets $E, F$ in the closure of a Euclidean domain $D$ is

$$
\operatorname{cap}(E, F ; D)=\inf _{u \in L} \int_{D}|\nabla u|^{n} d x
$$

where the infimum is taken over all functions in the class $L=L(E, F ; D)=\left\{u \in L^{1, n}(D) \cap\right.$ $\left.C(D \cup E \cup F):\left.u\right|_{E} \leq 0,\left.u\right|_{F} \geq 1\right\}$. Here $L^{1, n}(D)$ is the Sobolev space of locally integrable functions whose distributional derivatives are $n^{\text {th }}$-power integrable over $D$. An important property is that $\operatorname{cap}(E, F ; D)=\bmod (E, F ; D)$. These "capacity equals modulus" results follow, for instance, from [HK98, Proposition 2.17].

We now state some geometric estimates for conformal capacity which are based on the behavior of the family of all curves joining the sets $E, F$, and are mostly well-known. In this lemma and elsewhere in the paper,

$$
\Delta(E, F):=\operatorname{dist}(E, F) / \min \{\operatorname{diam}(E), \operatorname{diam}(F)\}
$$

is the relative distance between the pair $E, F$ of nondegenerate disjoint continua in $D$.

2.2. Lemma. Let $E, F$ be disjoint compact sets in $\mathbf{R}^{n}$.

(a) (Spherical Ring Estimate) If E, F are separated by the spherical ring $B(x ; s) \backslash \bar{B}(x ; t)$, then

$$
\operatorname{cap}\left(E, F ; \mathbf{R}^{n}\right) \leq \omega_{n-1}\left(\log \frac{s}{t}\right)^{1-n} .
$$

(b) If both $E$ and $F$ are connected, then

$$
\sigma_{n} \log (1+1 / \Delta(E, F)) \leq \operatorname{cap}\left(E, F ; \mathbf{R}^{n}\right) \leq \Omega_{n}(1+1 / \Delta(E, F))^{n} .
$$

(c) (Comparison Principle) If $A, B, E, F \subset D$ with $A, B$ also compacta, then

$$
\operatorname{cap}(E, F ; D) \geq 3^{-n} \min \{\operatorname{cap}(E, A ; D), \operatorname{cap}(F, B ; D), I\},
$$

where $I=\inf \{\operatorname{cap}(\alpha, \beta ; D) \mid \alpha \in \Gamma(E, A ; D), \beta \in \Gamma(F, B ; D)\}$.

(d) (Teichmüller Estimate) If $E, F$ are both connected, then for all $x, y \in E$ and $z, w \in F$

$$
\operatorname{cap}\left(E, F ; \mathbf{R}^{n}\right) \geq \tau\left(\frac{|x-z||y-w|}{|x-y||z-w|}\right)
$$

where $\tau(r)$ is the capacity of the Teichmüller ring $\mathbf{R}^{n} \backslash\left\{-1 \leq x_{1} \leq 0\right.$ or $\left.x_{1} \geq r\right\}$; i.e, $\tau(r)=\operatorname{cap}\left(\left[-e_{1}, 0\right],\left[r e_{1}, \infty\right] ; \mathbf{R}^{n}\right)$.

(e) There exists $\lambda=\lambda(n) \in\left[6,5 e^{(n-1) / 2}\right)$ such that when $E, F$ are both connected and $\Delta(E, F) \geq 1$,

$$
2^{1-n} \omega_{n-1}[\log (\lambda \Delta(E, F))]^{1-n} \leq \operatorname{cap}\left(E, F ; \mathbf{R}^{n}\right) \leq \omega_{n-1}[\log (\Delta(E, F))]^{1-n} .
$$

Proof. Some of these estimates can be found in [Vuo88]: specifically, (a) follows from (5.14), and Lemmas 5.35, 7.35, 7.38 give (c), (d), and the lower bound in (b) respectively.

The upper bound in (b) is well-known and easy to prove. Assuming $\delta=\operatorname{diam}(E) \leq$ $\operatorname{diam}(F)$, we choose any point $x \in E$ and define $\rho=1 / \operatorname{dist}(E, F)=1 / d$ in $B(x ; \delta+d)$. Then $\rho$ is easily seen to be admissible for $\left(E, F ; \mathbf{R}^{n}\right)$ and the estimate follows.

It remains to validate (e). Put $d=\operatorname{dist}(E, F), \delta=\min \{\operatorname{diam}(E), \operatorname{diam}(F)\}$ and assume that $d \geq \delta$. The upper bound is a consequence of (a) since $E, F$ are separated by every spherical ring $B(x ; d) \backslash \bar{B}(x ; \delta)$ with $x \in E$. 
To verify the lower inequality, we use the Teichmüller estimate (d) in conjunction with the inequality

$$
\tau(r) \geq \omega_{n-1} /\left(\log \lambda_{n}^{2}(1+r)\right)^{n-1}
$$

here $\lambda_{n} \in\left[4,2 e^{n-1}\right)$ and the inequality holds for all $r>1$; see [Vuo88, Lemma 7.22(2)].

Select points $x, y \in E$ and $z, w \in F$ with

$$
d=|x-z| \quad, \quad|x-y| \geq \delta / 2 \quad, \quad|z-w| \geq \delta / 2 .
$$

Using the triangle inequality $|y-w| \leq|y-x|+|x-z|+|z-w|$ we deduce that

$$
r=\frac{|x-z||y-w|}{|x-y||z-w|} \leq d\left(\frac{1}{|z-w|}+\frac{d}{|x-y||z-w|}+\frac{1}{|x-y|}\right) \leq 4 s(1+s)
$$

where $s=d / \delta=\Delta(E, F)$. Since $1+4 s(1+s) \leq 9 s^{2}$ when $s \geq 1$,

$$
\operatorname{cap}\left(E, F ; \mathbf{R}^{n}\right) \geq \tau(r) \geq \tau(4 s(1+s)) \geq \omega_{n-1} /\left(\log 9 \lambda_{n}^{2} s^{2}\right)^{n-1}
$$

as desired.

We require the following Localization Principle. We consider disjoint compact sets $E, F \subset$ $D \subset \mathbf{R}^{n}$ and for $r \geq \max \{\operatorname{diam}(E), \operatorname{dist}(E, F)\}$ we employ the notation $F_{r}=\overline{F \cap D_{r}}$ where $D_{r}$ is the component of $\{x \in D \mid \operatorname{dist}(x, E)<r\}$ containing $E$. Since the curve families $\left(E, F_{r} ; D_{r}\right)$ increase to $(E, F ; D)$, we know that $\operatorname{cap}\left(E, F_{r} ; D_{r}\right)$ increases to $\operatorname{cap}(E, F ; D)$ as $r$ tends to infinity. Here we provide a quantitative version of this fact.

2.3. Lemma. Let $E, F \subset D \subset \mathbf{R}^{n}$ be disjoint compacta. Given $0<a<b \leq \operatorname{cap}(E, F ; D)$, there exists a constant $C=C(a, b, n) \geq 1$ so that for all $r \geq C \operatorname{diam}(E)$, we have both $r \geq \operatorname{dist}(E, F)$ and

$$
\operatorname{cap}\left(E, F_{r} ; D_{r}\right) \geq a .
$$

Proof. Let $c_{1}:=(b+a) / 2$ and $c_{2}:=(b-a) / 2$, and put $\varepsilon:=\left(c_{1} / a\right)^{1 /(n-1)}-1$, so that $(1+\varepsilon)^{n-1} a=c_{1}$. We confirm that the constant $C=e^{L}$ works, where

$$
L=\max \left\{\left(\frac{\omega_{n-1}}{b}\right)^{\frac{1}{n-1}}, \frac{1}{\varepsilon}\left(2 \frac{\omega_{n-1}}{a} \frac{c_{1}}{c_{2}}\right)^{\frac{1}{n-1}}\right\}, \quad \text { so } \quad \frac{\omega_{n-1}}{L^{n-1}}=\min \left\{b, \frac{c_{2}}{2}\left(1+\varepsilon^{-1}\right)^{1-n}\right\} .
$$

Let us verify the assertions for $R=C \operatorname{diam}(E)$. Put $d=\operatorname{dist}(E, F)$ and $\delta=\operatorname{diam}(E)$. Thus $R=C \delta$. Fix any point $x \in E$ and let $A$ be the spherical ring $A=B(x ; R) \backslash \bar{B}(x ; \delta)$. If $R<d$, then $A$ strictly separates $E, F$ and so by Lemma 2.2(a)

$$
\operatorname{cap}(E, F ; D) \leq \operatorname{cap}\left(E, F ; \mathbf{R}^{n}\right)<\omega_{n-1} /\left(\log \frac{R}{\delta}\right)^{n-1}=\frac{\omega_{n-1}}{L^{n-1}} \leq b
$$

which contradicts the hypothesis that $\operatorname{cap}(E, F ; D) \geq b$.

Next, assume, for the sake of contradiction, that $\operatorname{cap}\left(E, F_{R} ; D_{R}\right)<a$. Then there is a $\left(E, F_{R} ; D_{R}\right)$-admissible density $\rho_{1}$ such that $a_{1}=\int_{D_{R}} \rho_{1}^{n}<a$. Select a density $\rho_{2}$ which is admissible for the spherical ring $A$ and satisfies $a_{2}=\int_{\mathbf{R}^{n}} \rho_{2}^{n}<\left(1+\varepsilon^{-1}\right)^{1-n} c_{2}$; such a $\rho_{2}$ exists by our definition of $A$ and $L$.

Extending $\rho_{1}$ to be zero outside $D_{R}$, it is readily verified that $\rho:=\rho_{1}+\rho_{2}$ is $(E, F ; D)$ admissible. A routine calculus exercise using the second derivative test provides the inequality

$$
\forall s, t, \varepsilon>0: \quad(s+t)^{n} \leq(1+\varepsilon)^{n-1} s^{n}+\left(1+\varepsilon^{-1}\right)^{n-1} t^{n},
$$


from which it follows that

$$
\int_{D} \rho^{n} \leq(1+\varepsilon)^{n-1} a_{1}+\left(1+\varepsilon^{-1}\right)^{n-1} a_{2}<c_{1}+c_{2}=b
$$

but this contradicts the assumption that $\operatorname{cap}(E, F ; D) \geq b$.

Many of our arguments are based on transferring capacity estimates between concentric balls, a process made possible by the next result; see [HK96, Lemma 3.2]

2.4. Fact. Suppose $B$ is a closed ball with $\sigma B \subset \bar{D}$ for some constant $\sigma>1$. Then for each $\vartheta \in(0,1]$, there is a constant $M=M(\vartheta, \sigma, n) \geq 1$ such that for all compacta $E \subset D \backslash \sigma B$,

$$
\operatorname{cap}(E, \vartheta B ; D) \leq \operatorname{cap}(E, B ; D) \leq M \operatorname{cap}(E, \vartheta B ; D) .
$$

In fact, we can take $M=(1+\log (1 / \vartheta) / \log (\sigma))^{n-1}$.

2.E. Capacity of Möbius Rings. Using the well-known formula for the capacity of a spherical ring, in conjunction with an appropriate Möbius transformation, we obtain the following useful formula for the capacity of a pair of disjoint balls. Lacking a precise reference for this, we record a proof.

2.5. Lemma. Assuming that

$$
d=\operatorname{dist}(B(x ; r), B(y ; s))=|x-y|-(r+s)>0,
$$

we have

$$
\operatorname{cap}\left(\bar{B}(x ; r), \bar{B}(y ; s) ; \mathbf{R}^{n}\right)=\omega_{n-1} \Phi(t)^{1-n}
$$

where

$$
t=\frac{d}{2 r s}(2 r+d+2 s)=\frac{|x-y|^{2}-(r+s)^{2}}{2 r s}
$$

and

$$
\Phi(t)=\log \left(1+t+\sqrt{(1+t)^{2}-1}\right) .
$$

Here $\Phi$ is increasing and satisfies

$$
\begin{aligned}
\log (1+t) & \leq \Phi(t) \leq \log (2[1+t]) \quad \text { for } t \geq 0 \\
\frac{2}{\sqrt{3}} \sqrt{t} & \leq \Phi(t) \leq \frac{2}{\sqrt{2}} \sqrt{t} \quad \text { for } 0 \leq t \leq 1 \\
\Phi(2 t) & \leq \sqrt{2} \Phi(t) \quad \text { for } t \geq 0 ;
\end{aligned}
$$

so, $\Phi(t)$ is comparable with $\sqrt{t}$ for $0<t \leq 1$, comparable with $\log (1+t)$ for $t \geq 1$, and enjoys a doubling property.

Proof. Select points $u, v, w, z$ on the line through $x, y$ so that

$$
d=|u-z| \quad, \quad 2 r=|u-v| \quad, \quad 2 s=|z-w| .
$$

Then

$$
\frac{|u-z||v-w|}{|u-v||z-w|}=\frac{d(2 r+d+2 s)}{2 r \cdot 2 s}=\frac{t}{2} .
$$

There is a Möbius transformation which maps $B(x ; r)$ to the unit ball, $\bar{B}(y ; s)$ to $\hat{\mathbb{C}} \backslash B(0 ; R)$ and sends the points $u, v, w, z$ to

$$
u^{\prime}=e=(1,0, \ldots, 0) \quad, \quad v^{\prime}=-e \quad, \quad w^{\prime}=-R e \quad, \quad z^{\prime}=R e
$$


respectively. Since Möbius transformations preserve cross-ratios, we have

$$
\frac{t}{2}=\frac{\left|u^{\prime}-z^{\prime}\right|\left|v^{\prime}-w^{\prime}\right|}{\left|u^{\prime}-v^{\prime}\right|\left|z^{\prime}-w^{\prime}\right|}=\frac{(R-1)^{2}}{4 R} .
$$

Thus $1+t+\sqrt{(1+t)^{2}-1}=R$ and therefore our capacity formula holds.

Since $\Phi^{\prime}(t)=1 / \sqrt{(1+t)^{2}-1}$, it is a routine exercise to validate the inequalities involving $\Phi$. To check the doubling property, consider $F(t)=\sqrt{2} \Phi(t)-\Phi(2 t)$. Then $F(0)=0$ and, since $2 \cdot 4 t(t+1) \geq 4 t(t+2), F^{\prime}(t) \geq 0$ for all $t \geq 0$.

Let us note a useful fact concerning the above formulae, writing $E=\bar{B}(x ; r), F=\bar{B}(y ; s)$, and $\delta=\Delta(E, F)$. First, it is easy to check that $2 \delta \leq t \leq 2 \delta(\delta+2) \leq 4 \delta \max \{\delta, 2\}$. Combining this observation with the comparability of $\Phi(t)$ to $\sqrt{t}$ for $0 \leq t \leq 1$ and to $\log (1+t)$ for $t \geq 1$ we deduce that

$$
\operatorname{cap}\left(E, F ; \mathbf{R}^{n}\right) \simeq \begin{cases}\Delta(E, F)^{-(n-1) / 2} & \text { when } \Delta(E, F) \leq 1, \\ {[\log (1+\Delta(E, F))]^{1-n}} & \text { when } \Delta(E, F) \geq 1 .\end{cases}
$$

for any disjoint closed balls $E$ and $F$ in $\mathbf{R}^{n}$. (Note that the estimate for $\Delta(E, F) \geq 1$ also follows from the bounds in part (e) of Lemma 2.2.)

We can use Lemma 2.5 to obtain a different sort of capacity transfer estimate as follows.

2.7. Lemma. Suppose $A, B$ are disjoint closed balls in $\mathbf{R}^{n}$ and let $A^{\prime} \subset A$ be the closed ball with $\operatorname{dist}\left(A^{\prime}, B\right)=\operatorname{dist}(A, B)$ and $\operatorname{diam}\left(A^{\prime}\right)=1 / 2 \operatorname{diam}(A)$. Then

$$
\operatorname{cap}\left(A^{\prime}, B ; \mathbf{R}^{n}\right) \leq \operatorname{cap}\left(A, B ; \mathbf{R}^{n}\right) \leq 2^{(n-1) / 2} \operatorname{cap}\left(A^{\prime}, B ; \mathbf{R}^{n}\right) .
$$

Proof. Writing $d=\operatorname{dist}(A, B), r=2 r^{\prime}=\operatorname{diam}\left(A^{\prime}\right)$ and $2 s=\operatorname{diam}(B)$ we find via Lemma 2.5 that

$$
\frac{\operatorname{cap}\left(A, B ; \mathbf{R}^{n}\right)}{\operatorname{cap}\left(A^{\prime}, B ; \mathbf{R}^{n}\right)}=\left(\frac{\Phi\left(t^{\prime}\right)}{\Phi(t)}\right)^{n-1}
$$

where

$$
t=\frac{d}{2 r s}(2 r+d+2 s) \text { and } \quad t^{\prime}=\frac{d}{2 r^{\prime} s}\left(2 r^{\prime}+d+2 s\right)
$$

Now

$$
\frac{t^{\prime}}{t}=2 \frac{u+1}{u+2} \quad \text { where } u=\frac{d+2 s}{r},
$$

so $t \leq t^{\prime} \leq 2 t$, and hence $\Phi(t) \leq \Phi\left(t^{\prime}\right) \leq \Phi(2 t) \leq \sqrt{2} \Phi(t)$ as desired.

We conclude this subsection with a 'bootstrap' technique.

2.8. Lemma. Suppose $D \subset \mathbf{R}^{n}$ and there are constants $\tau>0, a>0$ such that for all disjoint closed balls $A, B$ in $D$,

$$
\Delta(A, B) \geq \tau \quad \Longrightarrow \quad \operatorname{cap}(A, B ; D) \geq a \operatorname{cap}\left(A, B ; \mathbf{R}^{n}\right) .
$$

Then

$$
\Delta(A, B) \geq \tau / 2 \quad \Longrightarrow \quad \operatorname{cap}(A, B ; D) \geq b \operatorname{cap}\left(A, B ; \mathbf{R}^{n}\right),
$$

where $b=b(a, n)=a / 2^{(n-1) / 2}$. 
Proof. Assume $2 r=\operatorname{diam}(A) \leq \operatorname{diam}(B)=2 s$. Put $d=\operatorname{dist}(A, B)$ and suppose $\tau / 2 \leq$ $\Delta(A, B)=d / 2 r<\tau$. Let $A^{\prime} \subset A$ be the closed ball with $\operatorname{dist}\left(A^{\prime}, B\right)=d$ and $\operatorname{diam}\left(A^{\prime}\right)=r$. Then $\Delta\left(A^{\prime}, B\right)=d / r \geq \tau$, so Lemma 2.7 in conjunction with our hypothesis yields

$$
\operatorname{cap}(A, B ; D) \geq \operatorname{cap}\left(A^{\prime}, B ; D\right) \geq a \operatorname{cap}\left(A^{\prime}, B ; \mathbf{R}^{n}\right) \geq\left(a / 2^{(n-1) / 2}\right) \operatorname{cap}\left(A, B ; \mathbf{R}^{n}\right)
$$

as required.

2.F. The k-cap Condition. Suppose $C>0$ and $0<\lambda \leq 1 / 2$. A proper subdomain $D$ of $\mathbf{R}^{n}$ is a $(C, \lambda)$-k-cap domain provided

$$
\forall x, y \in D: \quad k(x, y) \geq 2 \quad \Longrightarrow \quad k(x, y)^{n-1} \operatorname{cap}(\lambda \bar{B}(x), \lambda \bar{B}(y) ; D) \leq C .
$$

This is the two-sided version of a condition introduced by the first author in [Buc04] to study quasiconformal images of Hölder domains. As explained on p.26 of that paper, a $(C, \lambda)$-k-cap condition implies a $\left(C_{1} C, \lambda^{\prime}\right)$-k-cap condition for some $C_{1}=C_{1}\left(\lambda, \lambda^{\prime}, n\right)$. We mainly consider the case $\lambda=1 / 2$, and call a $(C, 1 / 2)$-k-cap domain simply a $C$ - $k$-cap domain.

Note also that a condition in the reverse direction to the $\mathrm{k}$-cap condition holds in all domains; i.e., for $0<\lambda \leq 1 / 2$, there exists $c=c(\lambda, n)>0$ such that

$$
\forall x, y \in D: \quad k(x, y) \geq 2 \quad \Longrightarrow \quad k(x, y)^{n-1} \operatorname{cap}(\lambda \bar{B}(x), \lambda \bar{B}(y) ; D) \geq c .
$$

For instance, this last estimate is implicit in the proof of [HK96, Theorem 6.1]. Thus in a k-cap domain $D$, we have $\operatorname{cap}(\lambda \bar{B}(x), \lambda \bar{B}(y) ; D) \simeq k(x, y)^{1-n}$, with constants of comparison dependent only on $\lambda, n$, and the k-cap parameters.

Every uniform domain in $\mathbf{R}^{n}$ is a k-cap domain, and the class of $\mathrm{k}$-cap domains is invariant under quasiconformal mappings (with a quantitative change of parameter $C$ ). For proofs of these statements see [Buc04].

2.G. Spherical Distance. In parts of the proofs of Theorems 1.1 and 1.4, we require certain information concerning domains equipped with the spherical metric rather than the Euclidean metric. In particular, we assert that the classes of uniform, QED, Loewner, Gromov hyperbolic, and k-cap domains are all the same whether we use the spherical metric or the Euclidean one. Here we explain these ideas.

First, recall that the spherical distance $s(x, y)$ is simply the length of the shorter subarc of the great circle on $\hat{\mathbb{C}}=\partial S^{n}$ joining the points corresponding to $x, y$ under (equatorial) stereographic projection. Alternatively, $s(x, y)=2 \arcsin [q(x, y) / 2]$ where $q(x, y)$ is the chordal distance,

$$
q(x, y)= \begin{cases}\frac{2|x-y|}{\sqrt{1+|x|^{2}} \sqrt{1+|y|^{2}}} & \text { when } x \neq \infty \neq y, \\ \frac{2}{\sqrt{1+|x|^{2}}} & \text { when } x \neq \infty=y .\end{cases}
$$

That is, $q(x, y)$ is the Euclidean distance (in $\mathbf{R}^{n+1} \supset \partial S^{n}$ ) between the points corresponding to $x, y$ under stereographic projection. It is easy to see that the Euclidean and chordal cross ratios are identical. Since $q(x, y) \leq s(x, y) \leq(\pi / 2) q(x, y)$, we deduce that the Euclidean and spherical cross ratios are bilipschitz equivalent.

A routine exercise shows that the spherical modulus of any family of curves in $\mathbf{R}^{n}$ equals its Euclidean modulus. (The identity map $(D, d) \rightarrow(D, s)$ is conformal and the conformal modulus is a conformal invariant. :-) It therefore follows that a domain is spherically QED if and only if it is Euclidean QED. 
To see that the same is true for the Loewner condition, we appeal to a result of Brania and Yang [BY02] who showed that Loewner spaces can be characterized by a so-called controlled modulus condition. This latter concept is described in terms of cross ratios and it is straightforward to see that a spherical controlled modulus condition is equivalent to a Euclidean one. Thus a domain is spherically Loewner if and only if it is Euclidean Loewner.

In a manner entirely analogous to $\S 2$.B, we can define a spherical quasihyperbolic distance on any domain $D \subsetneq \mathbf{R}^{n}$ equipped with the spherical metric; see [BHK01, Chapter 2]. This spherical quasihyperbolic distance is always bilipschitz equivalent to the Euclidean quasihyperbolic distance; see [BB05]. It therefore follows that a domain is spherically Gromov (i.e., its spherical quasihyperbolization is Gromov hyperbolic) if and only if it is Euclidean Gromov; except for a quantitative change in the value of $\Delta$, it does not matter whether $k$ is the Euclidean or spherical quasihyperbolic metric since they are bilipschitz equivalent.

Next we explain why spherical uniformity is the same as in the Euclidean setting. One way to see this is to use the so-called Möbius cigars as explained in [Väi88, 2.7 and 2.10]. Again, as these are defined in terms of cross ratios, it is routine to validate our assertion.

We have two possible definitions of the k-cap condition for a domain $D \subsetneq \mathbf{R}^{n}$ depending on whether we view $D$ as a domain in Euclidean space or on the Riemann sphere. These definitions are equivalent up to a quantitative change in the parameter $C$, because capacity is a conformal invariant and the Euclidean and spherical quasihyperbolic distances are bilipschitz equivalent.

Every uniform domain in $\mathbf{R}^{n}$ or in $\hat{\mathbb{C}}$ is a k-cap domain, and the class of k-cap domains is invariant under quasiconformal mappings (with a quantitative change of parameter $C$ ). For proofs of these statements in the Euclidean case, see [Buc04]; the spherical variants are proved similarly. According to results in [BB03], proper subdomains of $\hat{\mathbb{C}}$ are (spherical) Gromov domains if and only if they satisfy a certain (spherical) slice condition which in turn implies the k-cap condition [Buc04]. Since the spherical quasihyperbolic distance is bilipschitz equivalent to the Euclidean quasihyperbolic distance, it follows that all Gromov domains in $\mathbf{R}^{n}$ are also k-cap domains.

\section{Domains SATISFying CAPACITy CONDitions}

In this section we study Loewner and QED-type conditions. These have the general form

$$
\operatorname{cap}(E, F ; D) \geq \Psi_{n}(E, F)
$$

where $E, F$ are disjoint continua in a domain $D \subset \mathbf{R}^{n}$ and the positive "function" $\Psi_{n}$ depends (in some way) only on $E, F$ and possibly the dimension $n$.

We call $D$ a $\psi$-QED domain if (3.1) holds with $\Psi_{n}(E, F)=\psi\left(\operatorname{cap}\left(E, F ; \mathbf{R}^{n}\right)\right)$ where $\psi:[0, \infty) \rightarrow[0, \infty)$ is a homeomorphism. The most important, and original, inequalities of this form are the $M-Q E D$ conditions corresponding to $\psi(t)=t / M$ for some constant $M \geq 1$. When we speak of a $Q E D$ domain or a $Q E D$ condition, we always mean an $M$-QED domain or an $M$-QED condition for some $M \geq 1$.

We call $D$ a $\phi$-Loewner domain, or simply a Loewner domain, if (3.1) holds with $\Psi_{n}(E, F)=$ $\phi(\Delta(E, F))$, where $\phi:(0, \infty) \rightarrow(0, \infty)$ is decreasing; see $\S 3$. B. By parts (b) and (e) of Lemma 2.2, every QED domain, and more generally every $\psi$-QED domain, is a Loewner domain. Implications in the converse direction are one of the main themes of this section.

We take this opportunity to mention a nice result due to Bonk and Kleiner, [?, Proposition 3.1], which is related to the problem of determining when some capacity inequality gives the 
full Loewner condition. Note that an infinite cylinder (see our Examples 4.2, 4.3) fails to satisfy their capacity hypothesis.

3.A. QED-type conditions. We say that $D \subset \mathbf{R}^{n}$ is a $\psi$-QED domain if $\psi:[0, \infty) \rightarrow$ $[0, \infty)$ is a homeomorphism and for all disjoint continua $E, F$ in $D$,

$$
\operatorname{cap}(E, F ; D) \geq \psi\left(\operatorname{cap}\left(E, F ; \mathbf{R}^{n}\right)\right) .
$$

Clearly, $\psi(t) \leq t$ is a necessary restriction on such $\psi$. However, while our definition asks that $\psi$ be a homeomorphism, it is straightforward to verify the following useful shortcut.

3.2. Lemma. Suppose $\psi:[0, \infty) \rightarrow[0, \infty)$ satisfies: $\psi(t) \rightarrow 0, \infty$ as $t \rightarrow 0, \infty$ respectively, and for each $\tau>0$, $\inf \{\psi(t): t \geq \tau\}>0$. Then there exists a homeomorphism $\xi:[0, \infty) \rightarrow$ $[0, \infty)$ which satisfies $\psi(t) \geq \xi(t)$ for all $t>0$.

The typical nonlinear functions $\psi$ that arise in the literature have the form $\psi_{p, M}(t)=$ $M^{-1} \min \left\{t^{p}, t^{1 / p}\right\}$ with $p, M \geq 1$, a condition we call $M$-QED ${ }^{p}$, or simply $M$-QED if $p=1$.

As in [HK96] we can consider the location of the continua $E, F$ as well as looking at special types of continua. In particular we can relax the $\psi$-QED inequality by requiring it to hold only for all disjoint closed balls (or just closed Whitney balls) to get the class $\psi$-QED (or $\psi-\mathrm{QED}_{\mathrm{wb}}$, respectively). To be precise, consider inequalities of the form

$$
\operatorname{cap}\left(\mu_{x} \bar{B}(x), \mu_{y} \bar{B}(y) ; D\right) \geq \psi\left(\operatorname{cap}\left(\mu_{x} \bar{B}(x), \mu_{y} \bar{B}(y) ; \mathbf{R}^{n}\right)\right)
$$

where $\mu_{x} \bar{B}(x)$ and $\mu_{y} \bar{B}(y)$ are disjoint, and $0<\mu_{x}, \mu_{y} \leq 1$. We say that $D \subset \mathbf{R}^{n}$ is a $\psi-\mathrm{QED}_{\mathrm{b}}$ domain if all such inequalities hold, and that it is a $(\psi, \mu)-\mathrm{QED}_{\mathrm{wb}}$ domain if such inequalities hold whenever $0<\mu_{x}=\mu_{y}=\mu<1$. When the parameters $M$ or $\mu$ are irrelevant, we drop them from this notation.

Every $a$-uniform domain in $\mathbf{R}^{n}$ is $M$-QED for some $M=M(a, n)$; this follows easily from Jones' extension result for Sobolev spaces [Jon81, Theorem 1] (see also the work [GLV79] of Gol'dshtein, Latfullin, Vodop'yanov). Also it is trivially true that

$$
\psi-\mathrm{QED} \Longrightarrow \psi-\mathrm{QED}_{\mathrm{b}} \Longrightarrow \psi-\mathrm{QED}_{\mathrm{wb}} \text {. }
$$

The converse of the first implication fails; see [HK96, Example 4.1] and Example 4.3. Below we prove that the second implication is reversible modulo a quantitative change in $\psi$.

Our next result actually implies Theorem 1.5. It says that the $\psi-\mathrm{QED}_{\mathrm{wb}}$ condition is self-improving in two ways: first, an inequality for fixed size Whitney balls implies a similar inequality for all balls in $D$, and second, the function $\psi$, which might a priori grow very slowly, can be assumed to grow linearly for $t \geq 1$. As a special case of this result, let us note that all $\mathrm{QED}_{\mathrm{wb}}$ domains are actually $\mathrm{QED}_{\mathrm{b}}$ domains, and more generally, all $\mathrm{QED}_{\mathrm{wb}}^{p}$ domains are $\mathrm{QED}_{\mathrm{b}}^{p}$ domains, $p \geq 1$.

3.3. Theorem. Suppose that $D \subsetneq \mathbf{R}^{n}$ is $(\psi, \mu)-Q E D_{w b}$. Then $D$ is $\psi^{\prime}-Q E D_{b}$ where $\psi^{\prime}$ depends only on $n, \mu$, and $\psi$. In fact, we can take $\psi^{\prime}(t)=c \psi(c t)$ for $0<t<1$ and $\psi^{\prime}(t)=$ ct for $t \geq 1$, where $c=c(n, \mu)>0$.

Proof. We break our proof into two parts, the first half of which is to prove the desired inequality for subWhitney balls. That is, we establish the desired capacity inequality for all (disjoint) balls of the form $E:=\mu_{x} \bar{B}(x)$ and $F:=\mu_{y} \bar{B}(y)$ where $0<\mu_{x}, \mu_{y} \leq \mu$.

Let us define $\mu^{\prime}:=(1+\mu) / 2, E^{\prime}:=\mu B(x), E^{\prime \prime}:=\mu^{\prime} B(x), F^{\prime}:=\mu B(y), F^{\prime \prime}:=\mu^{\prime} B(y)$, $d:=\operatorname{dist}(E, F)$, and $d^{\prime}:=\operatorname{dist}\left(E^{\prime}, F^{\prime}\right)$, so that $E \subset E^{\prime} \subsetneq E^{\prime \prime}$ and $F \subset F^{\prime} \subsetneq F^{\prime \prime}$. According to (2.6), $\operatorname{cap}\left(E, F ; \mathbf{R}^{n}\right)$ is large exactly when $\Delta(E, F)$ is small, and vice versa. Thus it suffices 
to prove a QED inequality for the triple $(E, F ; D)$ when $\Delta(E, F)$ is small, and a suitable $\psi^{\prime}$-QED inequality otherwise.

Our "small $\Delta(E, F)$ " case is when $E^{\prime \prime}$ and $F^{\prime \prime}$ overlap. Then $B(x) \cup B(y)$ is an $a$-uniform domain for some $a=a(\mu)$, and so $M$-QED for some $M=M(n, \mu)$. Thus

$$
\operatorname{cap}(E, F ; D) \geq \operatorname{cap}(E, F ; B(x) \cup B(y)) \geq M^{-1} \operatorname{cap}\left(E, F ; \mathbf{R}^{n}\right),
$$

as desired.

Suppose instead that $E^{\prime \prime}$ and $F^{\prime \prime}$ are disjoint. We proceed as in the proof of Proposition 3.5 in [HK96]. We consider three subcases. First, suppose $\mu_{x}$ and $\mu_{y}$ are both at least as large as $\mu / 2$. Then two applications of the transfer estimate Fact 2.4 produce

$$
\begin{aligned}
\operatorname{cap}(E, F ; D) \geq M^{-1} \operatorname{cap}\left(E, F^{\prime} ; D\right) \geq & M^{-2} \operatorname{cap}\left(E^{\prime}, F^{\prime} ; D\right) \geq \\
& \geq M^{-2} \psi\left(\operatorname{cap}\left(E^{\prime}, F^{\prime} ; D\right)\right) \geq M^{-2} \psi(\operatorname{cap}(E, F ; D))
\end{aligned}
$$

where $M=M(\mu, n)=[1+\log 2 / \log ((\mu+1) / 2 \mu))]^{n-1}$.

Second, suppose $\mu_{x}<\mu / 2 \leq \mu_{y}$. As above, we use the transfer estimate to see that $\operatorname{cap}(E, F ; D) \geq M^{-1} \operatorname{cap}\left(E, F^{\prime} ; D\right)$. Next, note that

$$
\operatorname{cap}\left(E, F^{\prime} ; \mathbf{R}^{n}\right) \leq \min \left\{\operatorname{cap}\left(E, \partial E^{\prime} ; \mathbf{R}^{n}\right), \operatorname{cap}\left(E^{\prime}, F^{\prime} ; \mathbf{R}^{n}\right)\right\} \leq C(n) .
$$

According to the Comparison Principle (Lemma 2.2(c)),

$$
\operatorname{cap}\left(E, F^{\prime} ; D\right) \geq 3^{-n} \min \left\{\operatorname{cap}\left(E, \partial E^{\prime} ; D\right), \operatorname{cap}\left(E^{\prime}, F^{\prime} ; D\right), I\right\}
$$

where

$$
I=\inf \left\{\operatorname{cap}(\alpha, \beta ; D) \mid \alpha \in \Gamma\left(E, \partial E^{\prime} ; D\right), \beta \in \Gamma\left(E^{\prime}, F^{\prime} ; D\right)\right\} .
$$

Each $\beta \in \Gamma\left(E^{\prime}, F^{\prime} ; D\right)$ contains a subcurve $\beta^{\prime} \in \Gamma\left(\partial E^{\prime}, \partial E^{\prime \prime} ; E^{\prime \prime}\right)$. Note that $\Delta\left(\alpha, \beta^{\prime}\right) \leq$ $4 \mu / \min \{\mu, 1-\mu\}$. Since $\mu^{\prime} B(x)$ is uniform, we deduce that

$$
\operatorname{cap}(\alpha, \beta ; D) \geq \operatorname{cap}\left(\alpha, \beta^{\prime} ; \mu^{\prime} B(x)\right) \geq N^{-1} \operatorname{cap}\left(\alpha, \beta^{\prime} ; \mathbf{R}^{n}\right)
$$

(where $N=N(n))$ and thus $I \geq c(\mu, n)>0$. Now

$$
\operatorname{cap}\left(E, \partial E^{\prime} ; D\right)=\operatorname{cap}\left(E, \partial E^{\prime} ; \mathbf{R}^{n}\right) \geq \operatorname{cap}\left(E, F ; \mathbf{R}^{n}\right) \geq \psi\left(\operatorname{cap}\left(E, F ; \mathbf{R}^{n}\right)\right) .
$$

Also,

$$
\operatorname{cap}\left(E^{\prime}, F^{\prime} ; D\right) \geq \psi\left(\operatorname{cap}\left(E^{\prime}, F^{\prime} ; \mathbf{R}^{n}\right)\right) \geq \psi\left(\operatorname{cap}\left(E, F ; \mathbf{R}^{n}\right)\right) .
$$

Thus,

$$
\operatorname{cap}(E, F ; D) \geq M^{-1} \operatorname{cap}\left(E, F^{\prime} ; D\right) \geq b \psi\left(\operatorname{cap}\left(E, F ; \mathbf{R}^{n}\right)\right)
$$

where $b=b(\mu, n)=\left(3^{n} M\right)^{-1} \max \{1, c / C\}$.

Finally, suppose both $\mu_{x}<\mu / 2$ and $\mu_{y}<\mu / 2$. Note that

$$
\operatorname{cap}\left(E, F ; \mathbf{R}^{n}\right) \leq \min \left\{\operatorname{cap}\left(E, \partial E^{\prime} ; \mathbf{R}^{n}\right), \operatorname{cap}\left(F, \partial F^{\prime} ; \mathbf{R}^{n}\right), \operatorname{cap}\left(E^{\prime}, F^{\prime} ; \mathbf{R}^{n}\right)\right\} \leq C(n) .
$$

Again by the Comparison Principle (Lemma 2.2(c)),

$$
\operatorname{cap}(E, F ; D) \geq 3^{-n} \min \left\{\operatorname{cap}\left(E, F^{\prime} ; D\right), \operatorname{cap}\left(F, \partial F^{\prime} ; D\right), J\right\} ;
$$

here, as above,

$$
J=\inf \left\{\operatorname{cap}(\alpha, \beta ; D) \mid \alpha \in \Gamma\left(F, \partial F^{\prime} ; D\right), \beta \in \Gamma\left(E, F^{\prime} ; D\right)\right\} \geq c(\mu, n) .
$$

Also, by our previous argument, $\operatorname{cap}\left(E, F^{\prime} ; D\right) \geq b \psi\left(\operatorname{cap}\left(E, F ; \mathbf{R}^{n}\right)\right)$. Thus once more we deduce the wanted inequality $\operatorname{cap}(E, F ; D) \geq c(\mu, n) \psi\left(\operatorname{cap}\left(E, F ; \mathbf{R}^{n}\right)\right)$. 
We have now verified the desired capacity inequality for all subWhitney balls. We proceed to the general case where $E:=\mu_{x} \bar{B}(x)$ and $F:=\mu_{y} \bar{B}(y)$ are an arbitrary pair of disjoint balls in $\bar{D}$. (Thus $0<\mu_{x}, \mu_{y} \leq 1$.) By changing the definition of $\psi$ if necessary, we now assume a $\psi$-QED inequality for all subWhitney balls. As above, it suffices to prove a suitable $\psi^{\prime}$-QED inequality for the triple $(E, F ; D)$ when $\Delta(E, F)$ is large, and a QED inequality otherwise.

Assume that $\operatorname{diam}(E) \leq \operatorname{diam}(F)$. Choose a closed ball $F^{\prime} \subset F$ with $\operatorname{dist}\left(E, F^{\prime}\right)=d:=$ $\operatorname{dist}(E, F)$ and $\operatorname{diam}\left(F^{\prime}\right)=\operatorname{diam}(E)$. Then $\Delta\left(E, F^{\prime}\right)=\Delta(E, F)$. Here our 'easy' case is when $\Delta(E, F) \geq 1 / 100$, for suppose this holds. Then $\Delta\left(E, F^{\prime}\right) \leq \Delta\left(\mu E, \mu F^{\prime}\right) \lesssim \Delta\left(E, F^{\prime}\right)$, and thus according to $(2.6)$,

$$
\operatorname{cap}\left(E, F ; \mathbf{R}^{n}\right) \simeq \operatorname{cap}\left(E, F^{\prime} ; \mathbf{R}^{n}\right) \simeq \operatorname{cap}\left(\mu E, \mu F^{\prime} ; \mathbf{R}^{n}\right),
$$

where the comparability constants depend only on $n$ and $\mu$. Therefore, it follows that

$$
\begin{aligned}
\operatorname{cap}(E, F ; D) \geq \operatorname{cap}\left(E, F^{\prime} ; D\right) & \geq \operatorname{cap}(\mu E, \mu F ; D) \geq \\
& \geq \psi\left(\operatorname{cap}\left(\mu E, \mu F ; \mathbf{R}^{n}\right)\right) \simeq \psi\left(\operatorname{cap}\left(E, F^{\prime} ; \mathbf{R}^{n}\right)\right) \simeq \psi\left(\operatorname{cap}\left(E, F ; \mathbf{R}^{n}\right)\right) .
\end{aligned}
$$

Now suppose instead that $\Delta(E, F)<1 / 100$. By translating, we may assume that $x=0$ and $E=\bar{B}(0 ; r)$; thus $r=\mu_{x} d(x)>50 d$. Note that by $(2.6)$,

$$
\operatorname{cap}\left(E, F ; \mathbf{R}^{n}\right) \simeq \Delta(E, F)^{(1-n) / 2} \simeq(d / r)^{(1-n) / 2} .
$$

Define $A$ to be the set of all $z \in E$ such that $|z| \geq r-2 d$ and $\operatorname{dist}(r z /|z|, F) \leq 2 d$. Thus $A \cap \partial E$ is an annular cap with radius comparable to $\sqrt{r d}$ and thickness $2 d$, and so the $n$-measure of $A$ is

$$
|A| \simeq d \cdot(r d)^{(n-1) / 2}
$$

Consider a closed ball $B$ of the form $\bar{B}(z ; \mu d), z \in A,|z|=r-d$. Let $H$ be the hyperplane equidistant from $E$ and $F$ (so $H$ is normal to the line segment $[x, y]$ ). Let $B^{\prime}$ be the reflection of $B$ across $H$. Then $B^{\prime} \subset F^{\prime} \subset F$ and $\operatorname{dist}\left(B, B^{\prime}\right) \leq 5 d$, so $\Delta\left(B, B^{\prime}\right) \leq 5 / 2 \mu$. By Lemma 2.5 (cf. (2.6)), $\operatorname{cap}\left(B, B^{\prime} ; \mathbf{R}^{n}\right) \geq c=c(\mu, n)>0$, and so by the first part of our proof,

$$
\operatorname{cap}\left(B, B^{\prime} ; D\right) \geq \psi(c) .
$$

Appealing to the Localization Principle (Lemma 2.3), we can select a constant $C \geq 6$, which depends only of $n$ and $\psi(c)$, such that

$$
\operatorname{cap}\left(B, B^{\prime} ; D^{\prime}\right) \geq \psi(c) / 2
$$

where $D^{\prime}$ is the component of $D \cap B(z ; C d)$ containing $B$.

The collection of all closed balls $B=\bar{B}(z ; \mu d), z \in A,|z|=r-d$ has the property that the dilated balls $K \cdot B$ cover $A$ provided $K>1 / \mu$. Fixing $K:=C / \mu$, we may select a subcollection $\left\{B_{i}=B\left(z_{i} ; \mu d\right) \mid 1 \leq i \leq m\right\}$ of the original balls which satisfy two criterion: the balls $K \cdot B_{i}$ are pairwise disjoint, and the balls $5 K \cdot B_{i}$ cover $A$. Then $m$ is comparable with the ratio of the volumes of $A$ and $B_{i}$, so

$$
m \simeq|A| /\left|B_{i}\right| \simeq d \cdot(r d)^{(n-1) / 2} / d^{n} \simeq \Delta(E, F)^{-(n-1) / 2} \simeq \operatorname{cap}\left(E, F ; \mathbf{R}^{n}\right) ;
$$

the comparability constants depend only on $n, \mu$ and $K=C / \mu$.

As in a previous paragraph, we reflect each $B_{i}$ across $H$ to obtain $B_{i}^{\prime} \subset F^{\prime} \subset F$. There is an associated domain $D_{i}$ (namely, the component of $D \cap K \cdot B_{i}$ containing $B_{i}$ ) with the 
property that $\operatorname{cap}\left(B_{i}, B_{i}^{\prime} ; D_{i}\right) \geq \psi(c) / 2$. Since the balls $K \cdot B_{i}$ are pairwise disjoint, we get

$$
\operatorname{cap}(E, F ; D) \geq \sum_{i=1}^{m} \operatorname{cap}\left(B_{i}, B_{i}^{\prime} ; D_{i}\right) \geq \frac{\psi(c)}{2} m \simeq \Delta(E, F)^{-(n-1) / 2} \simeq \operatorname{cap}\left(E, F ; \mathbf{R}^{n}\right) .
$$

\subsection{Corollary. Every $Q E D_{w b}$ domain is in fact $Q E D_{b}$.}

Our next main result follows; it is the crucial ingredient used to establish Theorem 1.1.

3.5. Theorem. A domain in $\mathbf{R}^{n}$ is uniform if and only if it is a $k$-cap domain and $\psi$ - $Q E D_{w b}$ where $\psi$ satisfies the growth condition $\lim _{u \rightarrow 0^{+}} e^{c / u} \psi\left(u^{n-1}\right)=\infty$ for all $c>0$. All parameters depend only on each other and the dimension $n$.

Proof. It is enough to establish the sufficiency of these conditions. We follow the proof of [HK96, Theorem A], but see also [Buc04, Theorem 2.8].

Assume that $D$ is $\psi-\mathrm{QED}_{\mathrm{wb}}$ and satisfies a $\left(C_{k}, \lambda\right)$-k-cap condition, where $\lambda:=1 / 3$. Let $x, y \in D$ and assume that

$$
d(x) \leq d(y)<|x-y|, \quad|x-y| \geq \frac{3 d(x)}{2}, \quad \text { and } \quad k(x, y) \geq 2 .
$$

Put $A_{z}=\lambda \bar{B}(z)$ for $z=x, y$ and note that $\operatorname{dist}\left(A_{x}, A_{y}\right)>\min \left\{\operatorname{diam}\left(A_{x}\right), \operatorname{diam}\left(A_{y}\right)\right\}$. It now follows, as in [HK96, p.346], that

$$
\operatorname{cap}\left(A_{x}, A_{y} ; \mathbf{R}^{n}\right) \geq C(\log r(x, y))^{1-n}
$$

where $C=C(n)=\omega_{n-1}(2[1+\log (3 \lambda(n) / 2) / \log (3 / 2)])^{1-n}$ and $\lambda(n)$ is the constant from Lemma 2.2(e).

Utilizing the above estimate together with the $\mathrm{k}$-cap and $\psi-\mathrm{QED}_{\mathrm{wb}}$ conditions we obtain

$$
k(x, y) \leq \vartheta(r(x, y)) \quad \text { where } \quad \vartheta(t)=\left[C_{k} / \psi\left(C / \log ^{n-1} t\right)\right]^{1 /(n-1)} .
$$

We take $c=(n-1) C^{\prime}$, where $C^{\prime}=C(n)^{1 /(n-1)}$, in our hypothesized growth condition, and then make the change of variable $t=e^{C^{\prime} / u}$ to deduce that $\lim _{t \rightarrow \infty} \vartheta(t) / t=0$. An appeal to Fact 2.1 confirms that $D$ is uniform.

Notice that the above argument only used the growth estimate on $\psi$ with the one constant $c=(n-1) C^{1 /(n-1)}$ and only requires the capacity inequalities

$$
\operatorname{cap}\left(3^{-1} \bar{B}(x), 3^{-1} \bar{B}(y) ; \mathbf{R}^{n}\right) \lesssim \operatorname{cap}\left(3^{-1} \bar{B}(x), 3^{-1} \bar{B}(y) ; D\right) \lesssim k(x, y)^{1-n}
$$

for appropriate points $x, y$ in $D$.

3.B. Loewner versus QED. We call $D \subset \mathbf{R}^{n}$ a $\phi$-Loewner domain if $\phi:(0, \infty) \rightarrow(0, \infty)$ and for all non-degenerate disjoint continua $E, F$ in $D$,

$$
\Delta(E, F) \leq t \Longrightarrow \operatorname{cap}(E, F ; D) \geq \phi(t) .
$$

We may (and do) always assume that the Loewner control function $\phi$ is a decreasing homeomorphism. Moreover, we have the following special case of [HK98, Theorem 3.6].

3.6. Fact. If $D$ is a Loewner domain in $\mathbf{R}^{n}$, then it is Loewner for some control function $\phi$ that is comparable with $\log (2 / t)$ for $t \leq 1$, and with $(\log 2 t)^{1-n}$ for $t \geq 1$. 
Combining the above fact with parts (b) and (e) of Lemma 2.2, we see that a Loewner domain certainly satisfies a QED inequality for all pairs $E, F$ with $\Delta(E, F) \geq 1$. We now examine the links between Loewner and QED-type conditions.

3.7. Proposition. The $\psi-Q E D$ and $\phi$-Loewner conditions are equivalent. This equivalence is quantitative in the sense that $\psi$ and $\phi$ depend only on each other and $n$.

Proof. We appeal repeatedly to the capacity estimates in parts (b), (e) of Lemma 2.2, so we abbreviate these as (b), (e) below. It is immediate from (b) and (e) that $\psi$-QED domains are Loewner. Suppose therefore that $D \subsetneq \mathbf{R}^{n}$ is $\phi$-Loewner, and let us prove a $\psi$-QED condition.

If $\Delta(E, F) \geq 1$, then by (e) we have

$$
\operatorname{cap}(E, F ; D) \geq \phi(\Delta(E, F)) \geq \psi_{1}\left(\operatorname{cap}\left(E, F ; \mathbf{R}^{n}\right)\right),
$$

where $\psi_{1}(t)=\phi\left(\exp \left[\left(\omega_{n-1} / t\right)^{1 /(n-1)}\right]\right)$. If $\Delta(E, F) \leq 1$, then $(\mathrm{b}) \operatorname{implies}$ that $\operatorname{cap}\left(E, F ; \mathbf{R}^{n}\right) \leq$ $\Omega_{n}(2 / \Delta(E, F))^{n}$, and so

$$
\operatorname{cap}(E, F ; D) \geq \phi(\Delta(E, F)) \geq \psi_{2}\left(\operatorname{cap}\left(E, F ; \mathbf{R}^{n}\right)\right),
$$

where $\psi_{2}(t)=\phi\left(2\left(\Omega_{n} / t\right)^{1 / n}\right)$.

By (b), $\Delta(E, F) \geq 1$ whenever $\operatorname{cap}\left(E, F ; \mathbf{R}^{n}\right) \leq C_{1}:=\sigma_{n} \log 2$, and $\Delta(E, F) \leq 1$ whenever $\operatorname{cap}\left(E, F ; \mathbf{R}^{n}\right) \geq C_{2}:=2^{n} \Omega_{n}$. Thus we deduce a $\psi$-QED condition, where

$$
\psi(t)= \begin{cases}\psi_{1}(t), & t<C_{1}, \\ \min \left\{\psi_{1}(t), \psi_{2}(t)\right\}, & C_{1} \leq t \leq C_{2}, \\ \psi_{2}(t), & t>C_{2} .\end{cases}
$$

It is clear from the above proof that the restricted versions of Loewner and $\psi$-QED, where we assume these conditions only for balls or Whitney balls, are similarly equivalent.

3.8. Corollary. Every domain which satisfies a Loewner condition for fixed size Whitney balls actually satisfies a Loewner condition for all disjoint balls.

Here is a surprisingly useful consequence of the above.

3.9. Corollary. Every Loewner or $\psi$-QED domain in $\mathbf{R}^{n}$ is in fact $\xi$-QED for some homeomorphism $\xi:[0, \infty) \rightarrow[0, \infty)$ which satisfies $\liminf _{t \rightarrow 0^{+}} e^{c / t} \xi\left(t^{n-1}\right)=\infty$ for all $c>0$.

Proof. Let $D \subset \mathbf{R}^{n}$ be $\psi$-QED. By Proposition 3.7, $D$ is $\phi$-Loewner, and we can choose $\phi$ as specified in Fact 3.6. Then from the proof of Proposition 3.7, we also have that $D$ is $\xi$-QED where

$$
\xi(t)=\psi_{1}(t)=\phi\left(\exp \left[\left(\omega_{n-1} / t\right)^{1 /(n-1)}\right]\right) \quad \text { for } t<C_{1}=\sigma_{n} \log 2 \text {. }
$$

Thus,

$$
\lim _{t \rightarrow 0^{+}} e^{c / t} \xi\left(t^{n-1}\right)=\lim _{t \rightarrow 0^{+}} e^{c / t} \psi_{1}\left(t^{n-1}\right) \simeq \lim _{t \rightarrow 0^{+}} e^{c / t} t^{n-1}=\infty,
$$

and so $\xi$ satisfies the desired growth condition.

Next we provide a sufficient condition for a domain to be $\mathrm{QED}_{\mathrm{b}}$. 
3.10. Theorem. Suppose a domain $D \subset \mathbf{R}^{n}$ has the property that there are constants $C>0$, $\tau>0$ such that whenever $A, B$ are disjoint balls in $D$ with $\Delta(A, B) \geq \tau$,

$$
\operatorname{cap}(A, B ; D) \geq C /(\log \Delta(A, B))^{n-1} .
$$

Then $D$ is $M-Q E D_{b}$ where $M=M(C, \tau, n)$. In particular, every Loewner domain is $Q E D_{b}$.

Proof. In view of Theorem 3.3, it suffices to verify that the domain $D$ is $(M, 1 / 2)-\mathrm{QED}_{\mathrm{wb}}$. By Lemma $2.2(\mathrm{e})$, the hypotheses imply that $\operatorname{cap}(A, B ; D) \geq\left(C / \omega_{n-1}\right) \operatorname{cap}\left(A, B ; \mathbf{R}^{n}\right)$, whenever $\Delta(A, B) \geq \tau^{\prime}:=\max \{\tau, 1\}$. Repeated applications of Lemma 2.8 provide, for each $\varepsilon>0$, a constant $c=c(\varepsilon, \tau, C)>0$ (e.g., if $\varepsilon=\tau^{\prime} / 2^{m}, c=\omega_{n-1} /\left(C 2^{m(n-1) / 2}\right)$ works) such that

$$
\Delta(A, B) \geq \varepsilon \quad \Longrightarrow \quad \operatorname{cap}(A, B ; D) \geq c \cdot \operatorname{cap}\left(A, B ; \mathbf{R}^{n}\right)
$$

for any pair of disjoint closed balls $A, B$ in $D$.

It therefore suffices to prove a QED-type inequality for $A=1 / 2 \bar{B}(x), B=1 / 2 \bar{B}(y)$ when $\Delta(A, B) \leq 1 / 8$. But then $B(x) \cup B(y)$ is a uniform domain, and such a QED-type inequality follows, with $M=M(n)$, as in the "small $\Delta(E, F)$ " case in the proof of Theorem 3.3.

Fact 3.6 tells us that Loewner domains satisfy the hypotheses, and so are $\mathrm{QED}_{\mathrm{b}}$.

3.C. Proofs of Introductory Theorems. Here we corroborate the results described in the introduction. Recall that a domain $D$ is c-linearly locally connected, abbreviated $c$-LLC, provided points in $D \cap B(x ; R)$ (respectively, in $D \backslash \bar{B}(x ; R)$ ) can be joined by a continuum in $D \cap B(x ; c R)$ (respectively, in $D \backslash \bar{B}(x ; R / c)$ ). Uniform, QED and Loewner domains all enjoy this property; see [GM85, Lemma 2.11] and [HK98, Theorem 3.13].

First we point out that Theorem 1.5 is an immediate consequence of

Theorem 3.3. Next, we confirm that Loewner domains are both $\mathrm{QED}_{\mathrm{b}}$ and $\psi$-QED.

Proof of Theorem 1.3. According to Theorem 3.10 and Proposition 3.7, every Loewner domain is both $\mathrm{QED}_{\mathrm{b}}$ and $\psi$-QED.

Now we demonstrate that Loewner implies QED under certain conditions. In part (a) of Theorem 1.4, we employ the spherical metric; the reader is encouraged to review $\S 2$.G.

Proof of Theorem 1.4. We first prove (a). Since $D$ is assumed to be a Loewner domain, it is also spherically Loewner and therefore (thanks to [HK98, Theorem 3.13]) also spherically LLC. Moreover, $D$ is a spherical Gromov domain too; thus $D$ is an LLC Gromov subdomain of $\hat{\mathbb{C}}$ and so it is spherically uniform by [BHK01, Proposition 7.12]. Finally, spherical uniformity is equivalent to Euclidean uniformity which implies Euclidean QED.

To prove (b), we first note that since $D$ is Loewner, it supports a $(1, n)$-Poincaré inequality [HK98, Theorem 5.12]. Next, since we are assuming that $N:=\mathbf{R}^{n} \backslash D$ has $n$-measure zero, [Kos99, Theorem C] says that $N$ is removable for Sobolev functions in $W^{1, n}$. Thus $N$ is a so-called NED set and therefore $D$ is in fact 1-QED.

Finally, we prove (c). Theorem 7.11 in [HK91] says in particular that under the given hypotheses, $D \subset \mathbf{R}^{2}$ is QED if and only if it is a quasicircle domain and there is a constant $c>0$ such that $\Delta(E, F) \geq c>0$ for each pair $E, F$ of distinct boundary components of $D$. Since $D$ is Loewner, it is LLC and hence a quasicircle domain by [GM85, Theorem 2.21]. The $\Delta(E, F) \geq c$ condition holds for any plane Loewner domain as we now demonstrate.

To this end, suppose $E, F$ are distinct components of $\partial D$. (If $D$ is simply connected, then it is a quasidisk and hence uniform.) Let $A, B$ be the components of $\hat{\mathbb{R}}^{2} \backslash D$ corresponding to $E, F$ respectively. Assume $\infty \notin B$ and put $R=\hat{\mathbb{R}}^{2} \backslash(A \cup B)$. Thanks to [HK91, 
Theorem 6.13], the desired relative distance inequality will follow provided we can exhibit constants $M, m$ such that for all disjoint closed $\operatorname{arcs} \alpha, \beta$ on $F=\partial B$,

$$
\operatorname{cap}\left(\alpha, \beta ; \mathbf{R}^{2}\right) \geq M \quad \Longrightarrow \quad \operatorname{cap}(\alpha, \beta ; R) \geq m .
$$

Note that the Loewner capacity inequality holds for disjoint continua in $\bar{D}$; see for example the proof of [HK90, Theorem 2.8] or [HK98, Remark 6.38]. Thus, if $\alpha, \beta$ are subarcs of $F$ with $\operatorname{cap}\left(\alpha, \beta ; \mathbf{R}^{2}\right) \geq M=4 \pi$, then $\Delta(\alpha, \beta) \leq 1$, so $\operatorname{cap}(\alpha, \beta ; R) \geq \operatorname{cap}(\alpha, \beta ; D) \geq m=\phi(1)$.

Finally, we establish our various characterizations for uniformity.

Proof of equivalence of (a),(b),(c) in Theorem 1.1. Since we know that uniform domains have all of the properties listed in (b), (c), it suffices to show that these two conditions imply uniformity. As discussed at the end of $§ 2 . G$, Gromov hyperbolicity implies a k-cap condition, so the fact that (b) implies uniformity is a consequence of Theorem 3.5 (since $\mathrm{QED}_{\mathrm{b}}$ means we have a linear control function $\psi$ ). Next, Loewner domains are QED $_{\text {wb }}$ (by Theorem 1.3 or Theorem 3.10), so by Corollary 3.9 and Theorem 3.5 it follows that (c) implies uniformity.

Example 4.2 is a domain which is Gromov, k-cap and satisfies a Loewner inequality for arbitrary disjoint closed balls, but is not uniform. Example 4.1 is a domain which is LLC with respect to arcs, quasiconvex, and satisfies the k-cap condition, but is not uniform. These illustrate that the mix of conditions in Theorem 1.1 is, in some sense, optimal.

Proof of equivalence of (a),(b),(c) in Corollary 1.2. This follows from Theorem 1.1 once we show that $D$ is Loewner and $\mathrm{QED}_{\mathrm{wb}}$. As $G$ is uniform, it is QED; since capacity and modulus 'ignore' removable sets, $D$ is also QED, and thus both Loewner and $\mathrm{QED}_{\mathrm{wb}}$.

The value of Corollary 1.2 is that uniformity is typically much easier to verify than the Gromov, k-cap, or weak slice conditions. It allows us to construct domains that fail these other conditions by, for instance, removing a countable set from a uniform domain in such a way that the double cone arc condition is destroyed for paths of quasiminimal length between certain pairs of points. For more on $W^{1, p}$ removability, see [Kos99] and the references therein.

\section{EXAMPLES}

Here we present examples to help clarify the hypotheses in our main theorems. Our first two examples address the various conditions appearing in Theorem 1.1. The last two examples reveal that the classes of $\psi$-QED domains are distinct for 'different' $\psi$.

4.1. Example. There exists a bounded planar domain $D$ which is LLC with respect to arcs, quasiconvex, and satisfies the k-cap condition, but is not uniform nor Loewner nor $\mathrm{QED}_{\mathrm{wb}}$ nor does it satisfy a weak-slice condition.

Proof. For each $i \in \hat{\mathbb{N}}$, put $a_{i}:=2^{-i}, r_{i}:=a_{i} / 16, \varepsilon_{i}:=r_{i}^{2}$, and let $h_{i}:\left[-2 r_{i}, 2 r_{i}\right] \rightarrow\left[\varepsilon_{i}, r_{i}\right]$ be the even function which is linear on $\left[0, r_{i}-\varepsilon_{i}\right]$, constant on $\left[r_{i}-\varepsilon_{i}, 2 r_{i}\right]$, and satisfies $h_{i}(0)=\varepsilon_{i}, h_{i}\left(r_{i}-\varepsilon_{i}\right)=r_{i}=h_{i}\left(2 r_{i}\right)$. Our domain $D$ is the union of the open unit square $(0,1)^{2}$ and the 'M-shaped decorations' $D_{i}=L_{i} \cup B_{i} \cup R_{i}$ where: $B_{i}$ is the bow-tie shaped region

$$
B_{i}:=\left\{z=(x, y):\left|x-a_{i}\right|<2 r_{i}, 1+r_{i}<y<1+r_{i}+h_{i}\left(x-a_{i}\right)\right\},
$$

and $L_{i}, R_{i}$ are the left-hand and right-hand connecting squares

$$
L_{i}=\left(a_{i}-2 r_{i}, a_{i}-r_{i}\right) \times\left[1,1+r_{1}\right] \quad, \quad R_{i}=\left(a_{i}+r_{i}, a_{i}+2 r_{i}\right) \times\left[1,1+r_{i}\right] .
$$


It is easy to see that each component of $\partial D$ is a $K$-quasicircle for some universal constant $K$; indeed, the inner boundary components are $2 r_{i} \times r_{i}$ rectangles and the outer boundary component is certainly bounded turning. Thus $D$ is LLC; in fact, it is clear that $D$ is LLC with respect to arcs and also quasiconvex.

The proof that $D$ enjoys a k-cap condition is a lengthy but straightforward case analysis very similar to that in the proof of [Buc03, Theorem 3.6]; we leave the details to the reader.

To see that $D$ is not Loewner, hence not uniform, we note that the relative distance of the outer boundary component versus the $i^{\text {th }}$ inner boundary component is comparable to $\varepsilon_{i} / r_{i}=r_{i}$ which tends to zero. Alternatively, we can check that points in $D_{i}$ close to, but on either side of, the bottleneck at $x=a_{i}$ cannot be joined by uniform arcs.

Finally, by Theorem 1.1, $D$ cannot be $\mathrm{QED}_{\text {wb }}$ nor can $D$ satisfy a weak slice condition.

We always have

$$
\mathrm{QED} \Longrightarrow \psi-\mathrm{QED} \Longleftrightarrow \text { Loewner } \Longrightarrow \mathrm{QED}_{\mathrm{wb}} \Longrightarrow \psi-\mathrm{QED}_{\mathrm{wb}} \Longleftrightarrow \text { Loewner }_{\mathrm{wb}}
$$

where the last condition means that the Loewner condition is assumed only for Whitney balls. We next exhibit examples which illustrate that the converses of the last two implications fail to hold. It remains open as to whether or not the first implication can be reversed.

4.2. Example. Let $B=B(0,1)$ be the unit ball in $\mathbf{R}^{n-1}, n>1$. The infinite cylinder $T:=B \times \mathbf{R}$ is a Gromov and k-cap domain which is Loewner with respect to balls with control function $\phi(t) \simeq t^{1-n}$ for large $t$ (and so it is $\psi$-QED $_{\mathrm{b}}$ for some $\psi$ ). However, this tube is not Loewner (since it is not LLC), and is not Loewner with respect to (Whitney) balls for any control function $\phi(t) \simeq(\log t)^{1-n}$ for large $t$ (and so it is not $\mathrm{QED}_{\mathrm{wb}}$ either).

Note that the above cylinder $T$ is quasiconformally equivalent to a half-space $H$; this was shown for $n=3$ in [Väi71, 16.5], and a similar mapping works in all dimensions. Since $H$ is uniform, it is Gromov and k-cap, and so $T$ inherits these properties. We leave the justification of the other properties and non-properties of $T$ to the reader.

In [HK96, Example 4.1], it was shown that an infinite tube with certain points removed is $\mathrm{QED}_{\mathrm{b}}$ but not QED; since this domain is not LLC, it is also not Loewner. By appropriately modifying that example we now show that the $\psi-\mathrm{QED}_{\mathrm{b}}$ classes are distinct for sufficiently different $\psi$. Note that by Theorem 3.3, it is behavior near 0 that accounts for the differences among different $\psi$-QED $\mathrm{B}_{\mathrm{b}}$ conditions.

4.3. Example. Suppose $\psi, \eta:[0, \infty) \rightarrow[0, \infty)$ are homeomorphisms satisfying

$$
\liminf _{t \rightarrow 0^{+}} e^{1 / t} \psi(t)=\infty \text {. }
$$

and

$$
\forall c>0: \quad \lim _{t \rightarrow 0^{+}} \frac{\eta(c t)}{\psi(t)}=\infty .
$$

For $c>0$, let $\psi_{c}(t)=c \psi(c t)$, and define $\eta_{c}$ similarly. Then there exists $c>0$ and a $\psi_{c}-\mathrm{QED}_{\mathrm{b}}$ domain $D$ that fails to be $\eta_{c^{\prime}}-\mathrm{QED}_{\mathrm{b}}$ for every $c^{\prime}>0$. In particular, there is a $\mathrm{QED}_{\mathrm{b}}^{q}$ domain that is not a $\mathrm{QED}_{\mathrm{b}}^{p}$ domain for any $1 \leq p<q$.

Proof. We consider $n=2$ and define $D:=G \backslash A$, where $G:=(-1,1) \times \mathbf{R}$, and $A \subset \mathbf{R}^{2}$ is a discrete set to be specified below. Let $\varepsilon_{j}:=\exp \left(-c_{0} / \psi^{-1}(1 / j)\right), j \in \hat{\mathbb{N}}$, where $c_{0}>0$ is chosen so that $\varepsilon_{1}=1 / 2$. For $x=\left(x_{1}, x_{2}\right) \in D$, let $N(x)$ be the least integer strictly greater 
than $\left|x_{2}\right|$. We claim that we can choose $A$ so that $d(x):=\operatorname{dist}(x, \partial D) \leq \varepsilon_{N(x)}$ for all $x \in D$ and, for all $j \in \hat{\mathbb{N}}$, there exists $x^{j}=\left(x_{1}^{j}, j-1\right) \in D$ such that $4 d\left(x^{j}\right) \geq \varepsilon_{N\left(x^{j}\right)}=\varepsilon_{j}$.

This claim is not entirely obvious for general $\psi$, so let us justify it. First, let $A_{1}:=P_{1} \times Q_{1}$, where $P_{1}=Q_{1} \subset[-1,1]$ is the coarsest partition of $[-1,1]$ that splits it into an even number of subintervals, all of length $l_{1} \leq \varepsilon_{1}=1 / 2$; thus $P_{1}=\{0, \pm 1 / 2, \pm 1\}$. Inductively, for $j>1$, let $A_{j}^{+}:=P_{j} \times Q_{j}^{+}$, where $P_{j} \subset[-1,1]$ is the coarsest refinement of $P_{j-1}$ that splits $[-1,1]$ into an even number of subintervals, all of length $l_{j} \leq \varepsilon_{j}$, and $Q_{j}^{+}$is the partition of $[j-1, j]$ into subintervals of length $l_{j}$. Let $A_{j}^{-}:=P_{j} \times Q_{j}^{-}$be the reflection of $A_{j}^{+}$in the $x_{1}$-axis. It is routine to deduce the claim for $A:=A_{1} \cup\left(\cup_{j=2}^{\infty} A_{j}^{+} \cup A_{j}^{-}\right)$; notice that the peculiar definition of $N(x)$ is to ensure that we get a lower bound of the required type for $d\left(x^{j}\right)$.

We now show that $D$ is a $\psi_{c_{0}}-Q E D$ domain. As in [HK96, Example 4.1], this follows if we prove the defining inequality for pairs of balls $E:=(1 / 2) \bar{B}(x), F:=(1 / 2) \bar{B}(y)$, where $|x-y|>10$, so let $x=\left(x_{1}, x_{2}\right)$ and $y=\left(y_{1}, y_{2}\right)$ be such a pair of points. Note that $\left|x_{2}-y_{2}\right|>9$. Without loss of generality, we assume that $d(x) \leq d(y)$. Let $L:=|x-y|$ and $r:=d(x)=\min \{\operatorname{diam}(E), \operatorname{diam}(F)\} / 2$, so that $4 \leq(L-2) / 2 r \leq \Delta(E, F) \leq L / 2 r$.

Writing $s:=\log (1 / r)$, we now prove the capacity estimates

$$
\operatorname{cap}\left(E, F ; \mathbf{R}^{2}\right) \simeq 1 / \log (L / r)=(s+\log L)^{-1}
$$

and

$$
\operatorname{cap}(E, F ; D) \simeq(s+L)^{-1} .
$$

Note that (4.6) follows immediately from Lemma 2.2(c). Countable sets such as $A$ make no difference to conformal capacity, since they are removable for $W^{1, p}$, so $\operatorname{cap}(E, F ; D)=$ $\operatorname{cap}(E, F ; G)$. Since $G$ is a simply connected planar domain, it is a k-cap domain. It is readily established that $k_{G}(x, y) \simeq s+L$, thus yielding (4.7).

Comparing (4.6) and (4.7), we see that a QED inequality follows for this data if $s \geq L$, so we may assume that $s \leq L$. Thus $\operatorname{cap}(E, F ; D) \simeq 1 / L$. Since also $1 /(s+\log L) \leq 1 / s$, the $\psi_{c_{0}}$-QED inequality for this data follows if we show that $\psi\left(c_{0} / s\right) \lesssim 1 / L$. By construction,

$$
\psi\left(c_{0} / s\right) \leq \psi\left(c_{0} / \log \varepsilon_{N(x)}^{-1}\right) \leq \psi\left(\psi^{-1}(1 / N(x))\right)<1 /\left|x_{2}\right| .
$$

Since $d(x) \leq d(y)$, it similarly follows that $\psi\left(c_{0} / s\right)<1 /\left|y_{2}\right|$. But

$$
10 \leq L \leq 2 \max \{|x|,|y|\} \leq 4 \max \left\{\left|x_{2}\right|,\left|y_{2}\right|\right\}
$$

and so $\psi\left(c_{0} / s\right)<4 / L$, as required.

Given $c^{\prime}>0$, it remains to prove that $D$ is not $\eta_{c^{\prime}}$ QED. Given $j \in \hat{\mathbb{N}}, j>1$, let $x^{j}=\left(x_{1}^{j}, j-1\right)$ and $y^{j}=\left(x_{1}^{j},-j+1\right) \in D$ be such that

$$
\frac{1}{4} \exp \left(-c_{0} / \psi^{-1}(1 / j)\right) \leq d\left(x^{j}\right)=d\left(y^{j}\right) \leq \exp \left(-c_{0} / \psi^{-1}(1 / j)\right)
$$

and let $E_{j}:=(1 / 2) \bar{B}\left(x_{j}\right), F_{j}:=(1 / 2) \bar{B}\left(y_{j}\right)$. Writing $s_{j}=\log \left(1 / d\left(x^{j}\right)\right)$, estimate (4.6) says that $\operatorname{cap}\left(E_{j}, F_{j} ; \mathbf{R}^{2}\right) \simeq 1 /\left(s_{j}+\log (2 j-2)\right)$. Taking $t=\psi^{-1}(1 / j)$ in $(4.4)$, we get

$$
\exists j_{0} \geq 2: \quad j \geq j_{0} \Longrightarrow \exp \left(1 / \psi^{-1}(1 / j)\right) \geq j,
$$

and so $s_{j} \simeq 1 / \psi^{-1}(1 / j) \geq \log j$ when $j \geq j_{0}$. Thus $\operatorname{cap}\left(E_{j}, F_{j} ; \mathbf{R}^{2}\right) \simeq \psi^{-1}(1 / j) \rightarrow 0$ as $j \rightarrow \infty$, and so (4.5) implies that $\lim _{j \rightarrow \infty} \bar{j} \cdot \eta\left(c^{\prime} \operatorname{cap}\left(E_{j}, F_{j} ; \mathbf{R}^{2}\right)\right)=\infty$. On the other hand, 
estimate (4.7) gives $\operatorname{cap}\left(E_{j}, F_{j} ; D\right) \simeq 1 /\left(s_{j}+2 j-2\right) \leq 1 / j$, for all $j \geq j_{0}$. Thus the $\eta_{c^{\prime}}$ QED inequality fails when $E=E_{j}, F=F_{j}$, and $j$ is sufficiently large.

\section{REFERENCES}

[BB03] Z.M. Balogh and S.M. Buckley, Geometric characterizations of Gromov hyperbolicty, Invent. Math. 153 (2003), 261-301.

[BB05] Sphericalization and flattening, preprint (2005).

[BH05] S. Buckley and D.A. Herron, Uniform domains and weak slice conditions in metric spaces, in preparation (2005).

[BHK01] M. Bonk, J. Heinonen, and P. Koskela, Uniformizing Gromov hyperbolic spaces, Astérisque. 270 (2001), 1-99.

[Buc03] S. Buckley, Slice conditions and their applications, Future Trends In Geometric Function Theory (Univ. Jyväskylä), vol. 92, Rep. Univ. Jyväskylä Dept. Math. Stat., 2003, RNC Workshop held in Jyväskylä, June 15-18, 2003, pp. 63-76.

[Buc04] S.M. Buckley, Quasiconfomal images of Hölder domains, Ann. Acad. Sci. Fenn. Ser. Math. 29 (2004), 21-42.

[BY02] A. Brania and S. Yang, Domains with controlled modulus and quasiconformal mappings, Nonlinear Stud. 9 (2002), no. 1, 57-73.

[CGN00] L. Capogna, N. Garofalo, and D-M Nhieu, Examples of uniform and NTA domains in Carnot groups, Proceedings on Analysis and Geometry (Novosibirsk), Izdat. Ross. Akad. Nauk Sib. Otd. Inst. Mat., 2000, (Novosibirsk Akad., 1999), pp. 103-121.

[CT95] L. Capogna and P. Tang, Uniform domains and quasiconformal mappings on the Heisenberg group, Manuscripta Math. 86 (1995), no. 3, 267-281.

[Geh87] F.W. Gehring, Uniform domains and the ubiquitous quasidisk, Jahresber. Deutsch. Math.-Verein 89 (1987), 88-103.

[GLV79] V.M. Gol'dshtein, T.G. Latfullin, and S.K. Vodop'yanov, Criteria for extension of functions of the class $l_{2}^{1}$ from unbounded plane domains, Siberian Math. J. 20 (1979), 298-301.

[GM85] F.W. Gehring and O. Martio, Quasiextremal distance domains and extension of quasiconformal mappings, J. Analyse Math. 45 (1985), 181-206.

[GO79] F.W. Gehring and B.G. Osgood, Uniform domains and the quasi-hyperbolic metric, J. Analyse Math. 36 (1979), 50-74.

[GP76] F.W. Gehring and B.P. Palka, Quasiconformally homogeneous domains, J. Analyse Math. 30 (1976), 172-199.

[Gre01] A.V. Greshnov, On uniform and NTA-domains on Carnot groups, Siberian Math. J. 42 (2001), no. 5, 851-864.

[Hei01] J. Heinonen, Lectures on analysis on metric spaces, Springer-Verlag, New York, 2001.

[HK90] D.A. Herron and P. Koskela, Quasiextremal distance domains and conformal mappings onto circle domains, Complex Variables 15 (1990), 167-179.

[HK91] _ Uniform, Sobolev extension and quasiconformal circle domains, J. Analyse Math. 57 (1991), 172-202.

[HK96] Conformal capacity and the quasihyperbolic metric, Indiana Univ. Math. J. 45 (1996), no. $2,333-359$.

[HK98] J. Heinonen and P. Koskela, Quasiconformal maps in metric spaces with controlled geometry, Acta. Math. 181 (1998), 1-61.

[Joh61] F. John, Rotation and strain, Comm. Pure Appl. Math. 14 (1961), 391-413.

[Jon81] P.W. Jones, Quasiconformal mappings and extendability of functions in Sobolev spaces, Acta Math. 147 (1981), 71-88.

[Kos99] P. Koskela, Removable sets for Sobolev spaces, Ark. Mat. 37 (1999), 291-304.

[MS79] O. Martio and J. Sarvas, Injectivity theorems in plane and space, Ann. Acad. Sci. Fenn. Ser. A I Math. $4(1978 / 79), 383-401$.

[Väi71] J. Väisälä, Lectures on n-dimensional quasiconformal mappings, Lecture Notes in Math., No. 229 (Berlin), Springer-Verlag, 1971.

[Väi88] _ Uniform domains, Tôhoku Math. J. 40 (1988), 101-118. 
[Väi10] , Free quasiconformality in Banach spaces II, Ann. Acad. Sci. Fenn. Ser. Math. 16 (1991), $255-310$.

[Vuo88] M. Vuorinen, Conformal geometry and quasiregular mappings, Lecture Notes in Math., No. 1319 (Berlin), Springer Verlag, 1988.

Department of Mathematics, National University of Ireland, Maynooth, Co. Kildare, IRELAND

E-mail address: sbuckley@maths.nuim.ie

Department of Mathematics, University of Cincinnati, OH 45221, USA

E-mail address: david.herron@math.uc.edu 